\title{
Evidências científicas acerca da assistência do enfermeiro ao idoso vítima de queda
}

\author{
Scientific evidence on the role of nurses in the elderly victim
}

Evidencias científicas acerca del papel del enfermero al anciano víctima de caída

Maria dos Milagres Santos da Costa르. José Newton Fernandes da Silva Júnior ${ }^{3}$, Ellen Eduarda Santos Ribeiro ${ }^{1 *}$, Kerolayne Laiz Barbosa de Morais ${ }^{1}$, Francinalva de Almeida ${ }^{2}$, Danielle Christina de Oliveira Santos², Kamilla Pinheiro Saraiva², Ana Claudia de Andrade Rezende ${ }^{4}$.

\section{RESUMO}

Objetivo: Descrever, por meio de evidências científicas, acerca da assistência do enfermeiro diante de pacientes idosos vítimas de quedas. Métodos: Trata-se de uma revisão integrativa da literatura, realizada nos meses de abril a outubro de 2017, desenvolvida em seis etapas segundo Mendes, Silveira e Galvão, com artigos nacionais disponíveis na integra indexados nas bases de dados LILACS, SCIELO e MEDLINE. Ao realizar o cruzamento dos três termos ("Idoso", "Trauma" e "Enfermagem"), foram encontradas 1068 publicações, entretanto apenas 50 estavam dentro dos critérios de inclusão e apenas cinco foram selecionados para compor a análise e a categorização propostas neste trabalho. Resultados: A análise da distribuição dos artigos possibilitou o agrupamento dos dados em duas categorias: Características e aspectos assistenciais do atendimento de enfermagem ao idoso traumatizado e Reabilitação e reintegração do idoso vítima de trauma. Conclusão: Observou-se que o enfermeiro, a partir da educação em saúde, pode melhorar os cuidados e as orientações com os pacientes idosos vítimas de queda.

Palavras-chave: Enfermagem, Idoso, Trauma.

\section{ABSTRACT}

Objective: To describe, through scientific evidence, the nurse's assistance to elderly patients who are victims of falls. Methods: This is an integrative review of the literature, carried out in April to October 2017, developed in six stages according to Mendes, Silveira and Galvão, with national articles available in the index LILACS, SCIELO and MEDLINE. When crossing the three terms ("Elderly", "Trauma" and "Nursing"), 1068 publications were found, however only 50 were within the inclusion criteria and only five were selected to compose the analysis and categorization proposed in this study. Results: The analysis of the distribution of articles made it possible to group the data into two categories: Characteristics and assistance aspects of nursing care for traumatized elderly people and Rehabilitation and reintegration of traumatized elderly people. Conclusion: It was observed that nurses, from health education, can improve care and guidance with elderly patients falling victims.

Keywords: Nursing, Elderly, Trauma.

\footnotetext{
${ }^{1}$ Discente do Departamento de Enfermagem pela Universidade Federal do Piauí, Teresina, Piauí.

${ }^{\star}$ E-mail: ellenribeirosr@gmail.com

${ }^{2}$ Graduação em Enfermagem pela Associação de Ensino Superior do Piauí, Teresina,Piauí.

${ }^{3}$ Graduação em Fisioterapia pela Universidade Estadual do Piauí, Mestrando de pós-graduação em Ciência de Saúde pela Universidade Federal do Piauí, Teresina, Piauí.

${ }^{4}$ Graduação em Enfermagem pelo Centro Universitário Uninovafapi, Teresina, Piauí.
}

SUBMETIDO EM: 11/2018

ACEITO EM: 12/2018

PUBLICADO EM: 4/2019

REAS/EJCH | Vol.Sup.22 | e578 | DOI: https://doi.org/10.25248/reas.e578.2019 Página 1 de 8 


\section{RESUMEN}

Objetivo: Describir, por medio de evidencias científicas, acerca de la asistencia del enfermero ante pacientes ancianos víctimas de caídas. Métodos: Se trata de una revisión integrativa de la literatura, realizada en los meses de abril a octubre de 2017, desarrollada en seis etapas según Mendes, Silveira y Galvão, con artículos nacionales disponibles en la integración indexados en las bases de datos LILACS, SCIELO y MEDLINE. AI realizar el cruce de los tres términos ("Anciano", "Trauma" y "Enfermería"), se encontraron 1068 publicaciones, sin embargo sólo 50 estaban dentro de los criterios de inclusión y sólo cinco fueron seleccionados para componer el análisis y la categorización propuestas en este trabajo. Resultados: El análisis de la distribución de los artículos posibilitó la agrupación de los datos en dos categorías: Características y aspectos asistenciales de la atención de enfermería al anciano traumatizado y Rehabilitación y reintegración del anciano víctima de trauma. Conclusión: Se observó que el enfermero, a partir de la educación en salud, puede mejorar los cuidados y las orientaciones con los pacientes ancianos víctimas de caída.

Palabras clave: Enfermería, Ancianos, Trauma.

\section{INTRODUÇÃO}

Segundo Ferretti et al. (2013), a sociedade brasileira no início do século XXI era considerada jovem, devido a alta taxa de natalidade e pelo seu recente surgimento. Entretanto, nos dias atuais, tal panorama se modifica, pois, a população passou a envelhecer, sendo que a expectativa de vida chegou a 73 anos. Diante disso, é necessário de um olhar holístico que busque sua longevidade e qualidade de vida. Essa busca permitiu uma preocupação maior com as quedas, tema bastante relevante na vida do idoso, haja vista que, o mesmo passa por modificações em seu físico, mental e social.

As quedas estão entre as principais causas de morbimortalidade nessa fase da vida do idoso. As contribuições para o aumento das quedas e dos traumas, são divididas em fatores extrínsecos e intrínsecos. Os fatores extrínsecos são aqueles relacionados ao ambiente em que o idoso se encontra, que inclui superfícies irregulares, pisos que são escorregadios, uma iluminação inadequada, posição postural, subir e descer escadas. Já os fatores intrínsecos estão relacionados ao perfil do próprio idoso tais, como a idade, a capacidade funcional, a presença de doenças crônicas, visão, as próprias limitações fisiológicas do organismo relacionado a envelhecimento (GAI J, GOMES L, NÓBREGA OT et al, 2010; MORSCH et al., 2016).

Nesse sentido, é importante entender sobre o processo multifatorial que envolve a queda. A pessoa senil passa por transformações que levam ao comprometimento da sua saúde como a redução da força muscular, a diminuição da agilidade e a perda do equilíbrio. Também, o decréscimo da capacidade do idoso em realizar as atividades instrumentais da vida diária, deixando-os mais dependentes e suscetíveis a ações que estão intimamente ligadas ao cair (ABDALA RP, JÚNIOR WB, JÚNIOR CRB et al, 2017).

É de extrema importância a realização de alguns cuidados especiais com os idosos, a exemplo de colocação de barra de ferro no interior das casas, retiradas de tapetes e proporcionar uma iluminação adequada, além de consultas periódicas com médicos geriatras, acompanhamento do enfermeiro na unidade básica, com a realização de visitas domiciliares, além da inclusão de toda equipe de saúde e família no processo de prevenção e recuperação de traumas, prevenindo assim, possível invalidez, imobilidade e restrição das atividades diárias, aumentando o bem estar e a qualidade de vida do idoso (FONSECA RSB, MOURA MEB, 2016).

Portanto, é de grande importância a atenção da Equipe de enfermagem a esses pacientes, desde a atenção primária, mantendo uma comunicação simples a fim de receber informações que possam levar a equipe a identificar e procurar solucionar através de conscientização da população através de campanhas , orientações que venham diminuir o número de idosos acometidos e traumatizados, e através do diálogo que se pode ter uma boa relação enfermeiro/paciente afim de compreender seus anseios e dificuldades, sua visão 
de mundo, sendo assim delimitou-se como questão norteadora: Quais são as evidências que a literatura traz a acerca dos cuidados de enfermagem diante de idosos vítimas de quedas?

Os enfermeiros precisam ser preparados para the dar com esses pacientes, uma das formas que 0 enfermeiro deve buscar para compreender os mesmos e buscando conhecer as habilidades, a história do paciente através e conversas, utilizar de meios como: escuta esclarecimento, orientação e desenvolvimento de habilidades capazes de melhorar a qualidade de vida desses pacientes junto a família e a comunidade como um todo (SILVA HC, PESSOA RL, MENEZES RMP et al, 2015).

Sendo assim, o presente estudo tem como objetivo avaliar o conhecimento e a produtividade cientifica acerca do papel do enfermeiro diante de pacientes idosos vítima de quedas, além de reunir evidencias científicas sobre essa assistência.

\section{METODOLOGIA}

Esse estudo trata-se de uma revisão integrativa, que tem o objetivo de realizar uma síntese do conhecimento de um conteúdo em específico, e com isso distinguir quais as perguntas precisam ser respondidas com a realização de novas pesquisas. Devido a isso, esse tipo de revisão, deve incluir análise de pesquisas importantes, que dão suporte para a tomada de decisão e a melhoria da prática clínica. Portanto é um método de pesquisa que admite a síntese de múltiplos estudos publicados e permite conclusões gerais sobre uma determinada área de estudo (MENDES KDS, SILVEIRA RCCP, GALVÃO CM et al, 2008).

De acordo com Souza et al. (2010); o aumento na quantidade e na complexidade de informações na área da saúde tornou imprescindível o desenvolvimento de artifícios, no contexto da pesquisa cientificamente embasada, capazes de delimitar etapas metodológicas mais concisas e de propiciar melhor utilização das evidências elucidadas em inúmeros estudos. Dessa forma, a revisão integrativa surge como uma metodologia que proporciona a síntese do conhecimento e a incorporação da aplicabilidade de resultados de estudos significativos na prática.

FIGURA 1 - Fluxograma de seleção de artigos que enfocam a produtividade cientifica acerca do conhecimento do enfermeiro diante dos pacientes idosos traumatizados, 2017.

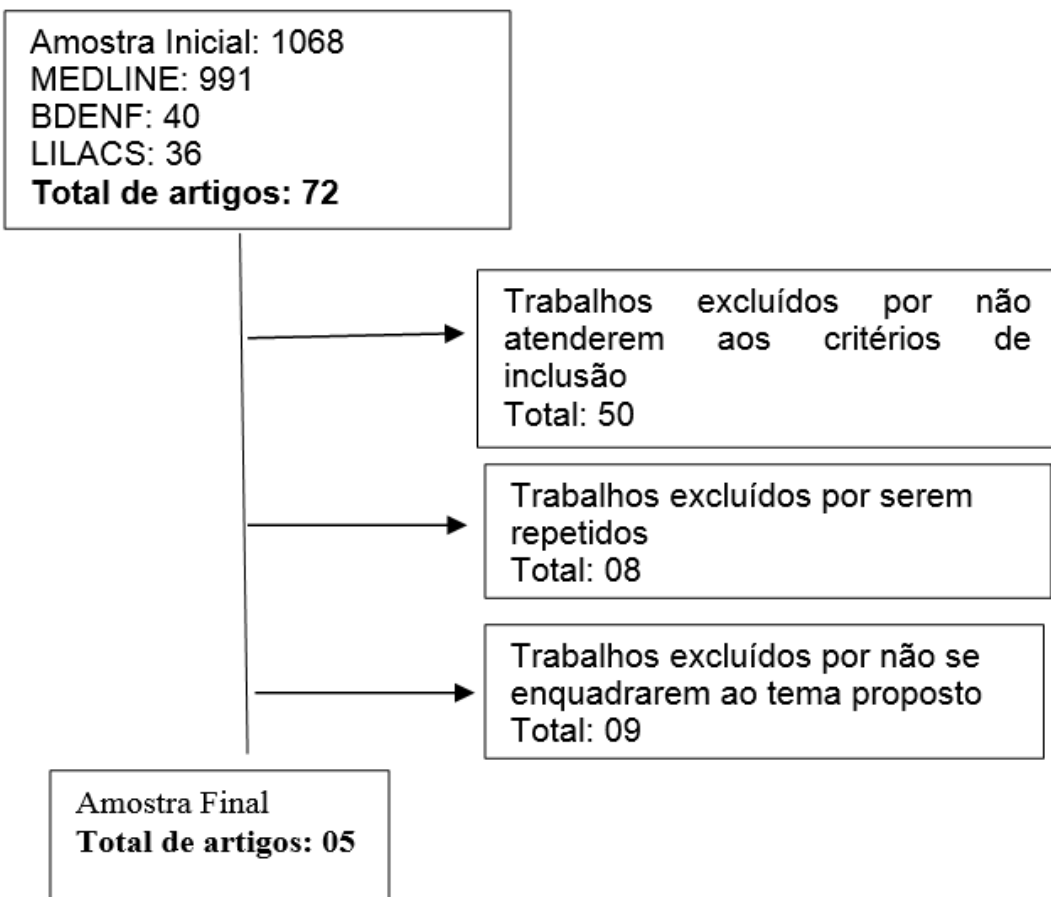

Fonte: Dados da pesquisa, 2018. 
Segundo Mendes et al.(2008); a revisão integrativa se desenvolve em seis etapas, que são: 1) identificar o tema e selecionar a hipótese ou questão de pesquisa para realizar a elaboração da revisão integrativa; 2) estabelecer quais são os critérios de inclusão e exclusão de estudos, amostragem ou busca na literatura; 3) definir quais as informações que serão extraídas dos estudos selecionados; 4) avaliar os estudos incluídos na revisão integrativa; 5) interpretar os resultados e por último, 6) apresentação da revisão/síntese do conhecimento.

Os dados foram coletados entre os meses de abril e outubro 2017, sendo utilizados os seguintes critérios de inclusão: artigos publicados no período de 2010 a 2017 sendo artigos em português e nacionais disponíveis na integra indexados nas bases de dados da Biblioteca Virtual em Saúde (BVS), sendo elas: LILACS, SCIELO E MEDLINE.

Foram utilizados como critérios de exclusão: artigos em inglês, artigos que não atendiam aos critérios do devido trabalho, artigos publicados em anos anteriores ou posteriores aos exigidos na dimensão temporal.

O acesso eletrônico teve como os sites periódicos Capes, utilizando para o levantamento dos artigos o agrupamento dos seguintes descritores cadastrados no DECS: "idoso", "trauma" e "enfermagem".

Para desenvolver a busca dos artigos, adotou-se pesquisar por descritores, seleção do período de publicação, seleção dos idiomas, leitura de títulos e resumos, logo após essa triagem, foi escolhido os que abordarem o tema, que atendam aos objetivos do estudo e posteriormente a exclusão dos que não atendam aos critérios descritos.

Os critérios de inclusão para a seleção da amostra foram: artigos completos publicados em português, com dimensão temporal entre 2010 a 2017 , abordando a temática da produtividade cientifica acerca do papel do enfermeiro frente a pacientes idosos traumatizados.

Os artigos foram numerados segundo a ordem de localização e a análise dos dados foi realizada na forma descritiva, proporcionando aos profissionais de diversas áreas avaliarem a qualidade das evidências.

Após a seleção e análise dos artigos, estes foram categorizados em uma tabela, com o objetivo de desenvolver uma melhor análise e estudo das publicações.

\section{RESULTADOS}

No cruzamento dos três descritores ("Idoso", "Trauma" e "Enfermagem") nas bases de dados selecionadas, foram encontradas 1068 publicações, dentre essas, 72 apresentavam texto na íntegra online. Foram excluídos 50 trabalhos, pois não atendem os dos critérios de inclusão, além disso, 8 por serem repetidos e 9 por não se enquadrarem no tema. Diante disso, foram selecionados cinco artigos para compor a análise e a categorização propostas neste trabalho.

Das cinco produções, 04 eram artigos quantitativos e 01 pesquisa documental, dos estudos quantitativos, dois eram do tipo corte transversal retrospectivo e dois descritivos. Os referidos estudos foram encontrados na Biblioteca Virtual em Saúde (BVS), que integra as principais bases de dados -SCIELO, LILACS, BDENF. Assim, o quadro abaixo apresenta uma síntese das principais características dos trabalhos incluídos na análise, indicando autores, o título da pesquisa, periódico indexado seus respectivos principais resultados obtidos. 
QUADRO 1 Distribuição dos artigos segundo autores, ano de publicação, Título do estudo, periódico indexado e principais resultados, Brasil, 2017.

\begin{tabular}{|c|c|c|c|}
\hline AUTOR, ANO & TITULO ARTIGO & PERIÓDICO & CONCLUSÃO \\
\hline $\begin{array}{l}\text { CAVALCANTE; } \\
\text { AGUIAR; } \\
\text { GURGEL, } 2012 .\end{array}$ & $\begin{array}{l}\text { Fatores associados a } \\
\text { quedas em idosos } \\
\text { residentes em um } \\
\text { bairro de Fortaleza, } \\
\text { Ceará }\end{array}$ & $\begin{array}{l}\text { Rev. Bras. } \\
\text { Geriatr. } \\
\text { Gerontol. }\end{array}$ & $\begin{array}{l}\text { Observou-se que } 42 \% \text { dos idosos apresentaram, no } \\
\text { mínimo, um episódio de queda nos últimos dois anos, } \\
\text { dos quais } 19 \% \text { se encontravam entre } 60 \text { e } 69 \text { anos, } \\
24 \% \text { entre } 70 \text { e } 79 \text { anos e } 57 \% \text { entre } 80 \text { e } 89 \text { anos. } \\
\text { As causas são relacionadas ao ambiente doméstico } \\
\text { inadequado (57\%). A maioria dos participantes ( } 80 \%) \\
\text { fazia uso de algum tipo de medicação prescrita por } \\
\text { médicos, sendo os anti-hipertensivos utilizados por } \\
42 \% \text { do grupo. A consequência mais comum das } \\
\text { quedas foi a fratura, indicada por } 43 \% \text { dos idosos, } \\
\text { tendo sido mais frequente a fratura de rádio (56\%). } \\
\text { Outras consequências citadas foram trauma craniano } \\
\text { (19\%), depressão (19\%) e ansiedade (19\%). } \\
\text { Necessitaram de internação hospitalar } 33 \% \text { dos } \\
\text { idosos vítimas de quedas. }\end{array}$ \\
\hline $\begin{array}{l}\text { PARREIRA, José } \\
\text { Gustavo et al., } \\
2014\end{array}$ & $\begin{array}{l}\text { Análise comparativa } \\
\text { das características do } \\
\text { trauma entre idosos } \\
\text { com idade superior e } \\
\text { inferior a } 80 \text { anos }\end{array}$ & Rev. Col. Bras. & $\begin{array}{l}\text { A idade variou de } 70 \text { a } 99 \text { anos, sendo maioria do } \\
\text { sexo masculino, sendo maior frequência de queda da } \\
\text { própria altura e menor frequência de fraturas de } \\
\text { membros superiores não observamos diferença } \\
\text { significativa na comparação das demais variáveis } \\
\text { entre os grupos.. }\end{array}$ \\
\hline $\begin{array}{l}\text { RODRIG; } \\
\text { CIOSAK, } 2012 .\end{array}$ & $\begin{array}{l}\text { Idosos vítimas de } \\
\text { trauma: análise de } \\
\text { fatores de risco }\end{array}$ & $\begin{array}{l}\text { Ver EscEnferm } \\
\text { USP }\end{array}$ & $\begin{array}{l}\text { A análise do estudo permitiu afirmar que, o gênero } \\
\text { feminino, a presença de cuidador, medicação de uso } \\
\text { contínuo e problemas auditivos aumentam } \\
\text { significativamente a probabilidade de trauma por } \\
\text { queda. Problemas de visão sem uso de óculos e } \\
\text { idosos com renda de até três salários mínimos } \\
\text { tendem a ter maior probabilidade de trauma por } \\
\text { queda. }\end{array}$ \\
\hline $\begin{array}{l}\text { SILVA; PESSOA; } \\
\text { MENEZES, } 2016 .\end{array}$ & $\begin{array}{l}\text { Trauma em idosos: } \\
\text { acesso ao sistema de } \\
\text { saúde pelo } \\
\text { atendimento pré- } \\
\text { hospitalar móvel }\end{array}$ & Rev. Latino-Am & $\begin{array}{l}\text { O estudo evidenciou uma prevalência causada por } \\
\text { quedas, acidentes de trânsito e violência, o que levou } \\
\text { ao predomínio das USB na prestação do primeiro } \\
\text { atendimento pelo serviço pré-hospitalar móvel. No } \\
\text { que tange às ações realizadas pela equipe de } \\
\text { enfermagem, ou com sua colaboração, no APH, } \\
\text { sobressaíram o controle circulatório, a abertura de } \\
\text { vias aéreas com controle cervical e a imobilização, e, } \\
\text { no que se refere ao acesso ao sistema de saúde por } \\
\text { meio do APH. }\end{array}$ \\
\hline $\begin{array}{l}\text { DEGANI, Gláucia } \\
\text { Costa et al.,2014. }\end{array}$ & $\begin{array}{l}\text { Idosas vítimas de } \\
\text { trauma: doenças } \\
\text { preexistentes, } \\
\text { medicamentos em } \\
\text { uso no domicílio e } \\
\text { índices de trauma }\end{array}$ & $\begin{array}{l}\text { REV Bras } \\
\text { Enfermagem. }\end{array}$ & $\begin{array}{l}\text { Foram estudados } 131 \text { idosos, média de idade } 69,9 \\
\text { anos, a maioria possuía doenças preexistentes e } \\
\text { metade deles usavam medicamentos no domicílio. } \\
\text { Houve representatividade de quedas (31,3\%), } \\
\text { seguidas por atropelamento (28,2\%), com } \\
\text { cabeça/pescoço sendo a região mais acometida } \\
(59,5 \%) \text {. Prevaleceu o trauma moderado }(44,3 \%) \text {, } \\
\text { com condições de sobrevida após o evento }(80,2 \%) \text {. } \\
\text { Houve associação entre mecanismo do trauma e } \\
\text { doença preexistente }(p=0,01) \text { e entre mecanismo do } \\
\text { trauma e sexo }(p=0,03) \text {. }\end{array}$ \\
\hline
\end{tabular}

Fonte: (Elaborado pela pesquisadora com base nos dados e resultados da pesquisa, 2017).

\section{DISCUSSÃO}

A análise da distribuição dos artigos por metodologia e instrumentos pesquisados possibilitou o agrupamento dos mesmos em duas categorias: Características e aspectos assistenciais do atendimento de enfermagem ao idoso traumatizado e Reabilitação e reintegração do idoso vítima de trauma. 


\section{Características e aspectos assistenciais do atendimento de enfermagem ao idoso traumatizado}

Parreira et al. (2013) evidencia que a idade dos idosos que são cometidos por quedas, varia de 70 a 79 anos de idade onde a maioria é representada por idosos do sexo masculino, isso é explicado pelo fato de estarem mais expostos aos riscos de acidentes de trânsito, de trabalho, quedas da própria altura e pelo modo de vida que estão inseridos. No entanto, segundo Landim et al. (2015) a maioria das internações de idosos é do sexo feminino, mulheres na faixa etária acima dos 60 anos de idade, pois tendem a ir com mais frequência aos hospitais para procurar tratamento e com isso, é feita a notificação da queda.

Segundo Biazin e Rodrigues (2009) as principais causas de queda em idosos estão relacionadas aos fatores extrínsecos e intrínsecos. As relacionadas aos fatores extrínsecos, estão: utilização de sapatos inadequados para a idade, escadas sem corrimão, pisos escorregadios, falta de iluminação adequada nos ambientes domésticos, camas altas, degraus autos, ou estreitos, tapetes soltos, vasos sanitário baixo, ausência de barra de suporte, cadeiras instáveis, via pública mal conservada com buracos ou irregulares, aliado a falta de conhecimento dos familiares e cuidadores quanto às medidas de prevenção de quedas.

No que se concerne aos fatores intrínsecos das sequelas destacam-se aqueles relacionados a própria idade como por exemplo: tontura, perda da capacidade visual, consumo de uso variado de medicação continua, declínio cognitivo, presença de doenças crônicas, pior desempenho físico, labirintite (BIAZIN DT, RODRIGUES RAP, 2009).

Por conseguinte, segundo Parreira et al. (2013) observou-se que 54 \% dos idosos apresentam no mínimo um episódio de queda. Em consonância desses autores, um outro estudo mostra que há um aumento nos eventos traumáticos que envolvem idosos, expondo eles a sua incapacidade funcional parcial ou total, porém mesmo ocorrendo um grande aumento desses fatores ainda existe muito descaso com os mesmos, ainda são poucos os estudos relacionados ao assunto abordado, como exemplo o que pode causar esses traumas e principalmente como preveni-los (CAMPOS JFS et al, 2007).

Diante desse grande problema e com tendência a piora progressiva devido ao envelhecimento populacional, dizem que os fatores que mais interferem no trauma em idosos, podem ser avaliados durante a consulta de enfermagem, afim de possibilitar ao profissional ações de saúde para a sua prevenção (PARREIRA JG et al, 2013; RODRIGUES J, CIOSAK SI, 2012).

Criar atividades que possam desenvolver e capacitar os cuidadores para que os mesmos possam colocar em prática ações que venham favorecer uma qualidade devida adequada ao idoso, implementar políticas socais e de saúde local condizentes com a realidade dos idosos (LANDIM ACF et al. 2015).

Para Parreira et al. (2013) a equipe de enfermagem pode atuar com medidas voltadas a prevenção, ainda na Atenção Básica para que essas estatísticas possam ser modificadas, através da identificação dos fatores de riscos físicos e ambientais, afim de adapta-los ou modifica-los, diminuindo assim os fatores que contribuem para a queda presentes nos idosos.

Diante disso, o enfermeiro para sistematizar a assistência de enfermagem ao idoso de uma forma eficaz, deverá utilizar sempre dos meios como: palestras, orientações, visitas domiciliares, além disso, deve tratar de possíveis patologias relacionadas, sempre identificando os fatores de risco que estão tanto no ambiente como no cotidiano do idoso.

E importante orienta-los a adotar atitudes saudáveis que previnam quedas nessa idade, realizar atividades físicas, consultas para avaliação dos níveis de pressão arterial (PA), uso de medicamentos e cuidados com o ambiente em que vive. Além disso, o enfermeiro também pode atuar na identificação precoce de todas as pessoas que se encontram em risco potencial de sofrer uma lesão por quedas, realizar visitas domiciliares, educação e saúde com ênfase no autocuidado, avaliar o perfil de risco á nível individual e coletivo (LANDIM ACF et al. 2015).

\section{Reabilitação e reintegração do idoso vítima de trauma}


É interessante que o profissional enfermeiro atue sempre buscando uma assistência de qualidade a esses pacientes, a fim de encontrar novos elementos e práticas que possam promover uma reabilitação, física, psicológica e social; procurando sempre fazer com que o idoso permaneça autônomo e independente, praticando atividades físicas que possam promover sua qualidade de vida dentro dos seus limites, encorajar a adaptação do ambiente para que se torne seguro e confortável, estimula-lo e inseri-lo em seu contexto social, prevenir os agravos (LANDIM ACF et al. 2015).

Para Ribeiro e Barter (2010) a reinserção social é uma diretriz preconizada nas principais políticas dirigidas ao idoso e também um dos objetivos da reabilitação. Sendo assim, gestores e profissionais reconhecem que o idoso requer mais atenção e tratamento diferenciado devido às suas especificidades. Este mesmo estudo finaliza explanando que poucos estão preparados para cuidar de idosos; os profissionais precisam se capacitar mais.

Para Landim et al. (2015) é importante a atuação do enfermeiro nas instituições de longa permanência para idosos desenvolvendo ações relacionadas a promoção de saúde, proteção, reabilitação e educação em saúde, promovendo a autonomia dos idosos em condições de dependência e oferecendo-lhes uma melhor qualidade de vida.

A partir de um outro estudo, evidencia-se uma participação ativa dos idosos na sociedade, onde a qual tem surgido na literatura com o termo "super idosos" (superelderly), ou "muito idosos", representado por indivíduos com mais de 80 anos, onde acredita-se que este subgrupo de doentes apresente um maior comprometimento da reserva orgânica funcional mesmo antes de sofrer um trauma, o que necessita atenção especial tanto do ponto de vista diagnóstico quanto terapêutico. Sendo assim, em uma outra vertente não tem muitos estudos que abordam as características específicas dos traumatizados "muito idosos", e, tampouco, comparam as lesões encontradas com idosos com idade superior e inferior a 80 anos.

Por conseguinte, segundo Cavalcante et al. (2012) quando se objetiva a reintegração do idoso vítima de quedas ou não, se faz necessárias ações preventivas na atenção primária à saúde, sobretudo em relação à oferta de atividades físicas que certamente contribuiriam com a redução do risco de quedas. Deve-se reforçar a importância do autocuidado e alertar as famílias e cuidadores para que participem da prevenção de queda com os idosos. Outro aspecto relevante é adaptar a infraestrutura dos domicílios, objetivando reduzir a ocorrência de quedas.

De acordo com Silva et al. (2016); em se tratar de um problema emergente de saúde, é necessário que haja uma melhor discursão e/ou compreensão do trauma na população idosa, para que a equipe multiprofissional, principalmente a enfermagem, faça um planejamento de estratégias e de implementações para assistência geriátrica mais específica e, consequentemente, contribuir para a redução das chances de sequelas temporárias ou permanentes.

\section{CONSIDERAÇÕES FINAIS}

Por conseguinte, a queda é um fator intimamente ligado ao envelhecer. A pessoa no seu processo de envelhecimento passa por transformações biopsicossociais e que muitas vezes podem causam intenso sofrimento, devido a debilidade do momento. Sua vida necessita de adequação para que o mesmo possa se reinserir e participar de suas atividades básicas e instrumentais diárias. Com isso, a equipe em saúde, dentre eles o enfermeiro, deve utilizar da escuta/esclarecimento para planejar uma assistência adequada a esses pacientes, orientando-os, buscando trabalhar de uma forma que atenda não apenas o idoso, mas a família e a comunidade como um todo, afim de desenvolver intervenções preventivas, preservando assim a independência e a qualidade de vida dos idosos. 


\section{REFERÊNCIAS}

1. ABDALA RP, JÚNIOR WB, JÚNIOR CRB et al. PADRÃO DE MARCHA, PREVALÊNCIA DE QUEDAS E MEDO DE CAIR EM IDOSAS ATIVAS E SEDENTÁRIAS. Rev Bras Med Esporte, São Paulo, v. 23, n. 1, p.26-30, 2017

2. BIAZIN DT, RODRIGUES RAP. Perfil dos idosos que sofreram trauma em Londrina - Paraná. Rev.Esc. enferm USP, v.43, n.3, p.602-8, 2009.

3. CAMPOS JFS, POLETTI NAA, RODRIGUES CDS et al. Trauma em idosos atendidos no pronto atendimento da emergência do Hospital de Base. Arq Ciênc. Saúde, v.14, n.4, p.193-7, 2007.

4. CAVALCANTE ALP, AGUIAR JB, GURGEL LA. Fatores associados a quedas em idosos residentes em um bairro de Fortaleza, Ceará. Rev. bras. geriatr. gerontol., Rio de Janeiro, v. 15, n. 1, p. 137-146, 2012.

5. FERRETTI F, LUNARDI D, BRUSCHI L. Causas e consequências de quedas de idosos em domicílio. Fisioterapia em Movimento, [s.I.], v. 26, n. 4, p.753-762, dez. 2013. FapUNIFESP (SciELO). http://dx.doi.org/10.1590/s010351502013000400005.

6. FONSECA RSB, MOURA MEB. Fatores de risco para quedas em idosos no domicílio. R. Interd, Teresina, v. 9, n. 2, p.206-215, 2016

7. GAI J, GOMES L, NÓBREGA OT et al., Fatores associados a quedas em mulheres idosas residentes na comunidade. Rev Assoc Med Bras, Brasilia, v. 3, n. 56, p.327-332, 2010

8. LANDIM ACF, PINHEIRO FM; PESSANHA FS et al, Assistência de enfermagem a idosos com traumas ósseos: uma revisão integrativa. Revista de Pesquisa Cuidado é Fundamental Online, Rio de Janeiro, v. 7, n. 1, p.20832103, 2015

9. MENDES KDS, SILVEIRA RCCP, GALVÃO CM. Revisão integrativa: método de pesquisa para a incorporação de evidências na saúde e na enfermagem. Texto Contexto e Enfermagem. v. 17, n. 4, 2008.

10. MORSCH $P$ et al. A problematização da queda e a identificação dos fatores de risco na narrativa de idosos. Ciência \& Saúde Coletiva, [s.l.], v. 21, n. 11, p.3565-3574, nov. 2016. FapUNIFESP (SciELO). http://dx.doi.org/10.1590/1413-812320152111.06782016.

11. NASCIMENTO, Janaína Santos; TAVARES, Darlene Mara dos Santos. PREVALÊNCIA E FATORES ASSOCIADOS A QUEDAS EM IDOSOS. Texto \& Contexto - Enfermagem, [s.I.], v. 25, n. 2, p.1-9, 2016. FapUNIFESP (SciELO). http://dx.doi.org/10.1590/0104-07072016000360015.

12. PARREIRA JG, FARRATH S SOLDÁ SC et al, Análise comparativa das características do trauma entre idosos com idade superior e inferior a 80 anos. Rev. Col. Bras. Cir., São Paulo, v. 4, n. 40, p.269-274, 2013.

13. RIBEIRO AP, BARTER EACP. Atendimento de reabilitação à pessoa idosa vítima de acidentes e violência em distintas regiões do Brasil. Ciência \& Saúde Coletiva, [s.i], v. 6, n. 15, p.2729-2740, 2010.

14. RODRIGUES J, CIOSAK SI. Idosos vítimas de trauma: análise de fatores de risco. Rev. esc. enferm. USP, São Paulo, v. 46, n. 6, p. 1400-1405, Dec. 2012.

15. SILVA HC, PESSOA RL, MENEZES RMP. Trauma in elderly people: access to the health system through prehospital care. Rev. Latino-Am. Enfermagem, v.24, n.2690, 2016.

16. SILVA JPG, COSTA KNFM, SILVA GRF et al,. Consulta de enfermagem a idosos: instrumentos da comunicação e papéis da enfermagem segundo Peplau. Esc Anna Nery, [s.i], v. 19, n. 1, p.154-161, 2015.

17. SOUZA MT, SILVA MD, CARVALHO R. Revisão integrativa: o que é e como fazer. Einstein. v. 8, n. 1.p. 102106, 2010. 